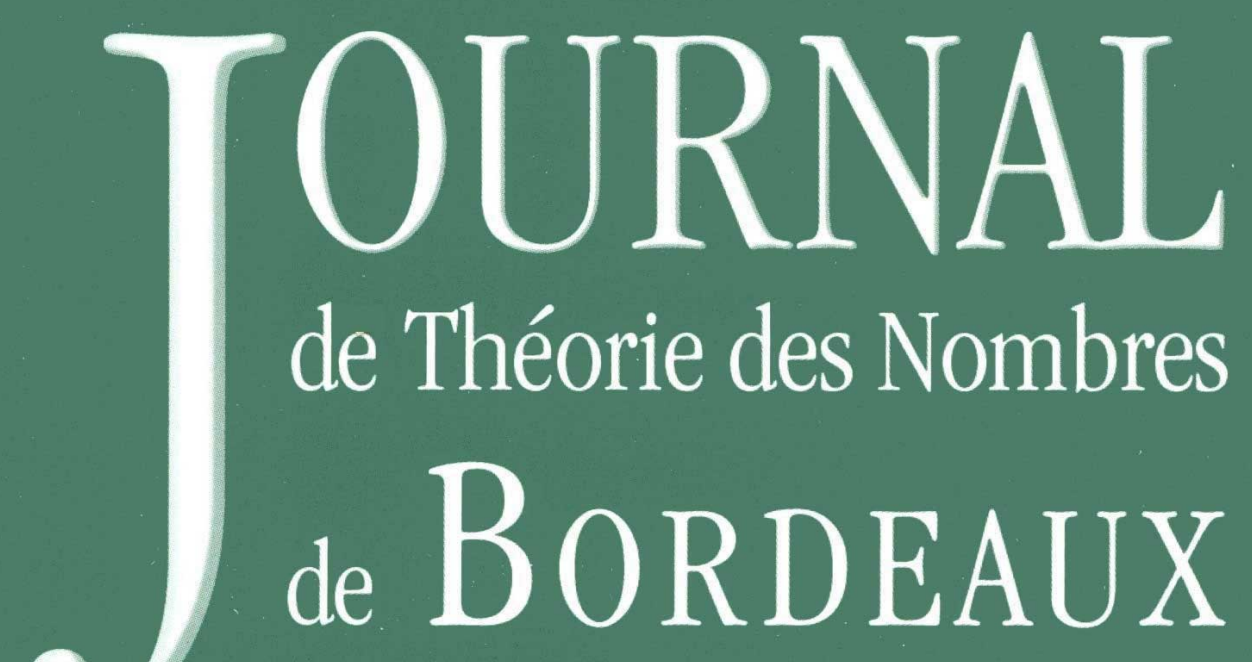

anciennement Séminaire de Théorie des Nombres de Bordeaux

Dan YASAKI

\title{
Perfect unary forms over real quadratic fields
}

Tome 25, nº 3 (2013), p. 759-775.

<http://jtnb.cedram.org/item?id=JTNB_2013__25_3_759_0>

(C) Société Arithmétique de Bordeaux, 2013, tous droits réservés.

L'accès aux articles de la revue «Journal de Théorie des Nombres de Bordeaux » (http://jtnb.cedram.org/), implique l'accord avec les conditions générales d'utilisation (http://jtnb.cedram. org/legal/). Toute reproduction en tout ou partie de cet article sous quelque forme que ce soit pour tout usage autre que l'utilisation à fin strictement personnelle du copiste est constitutive d'une infraction pénale. Toute copie ou impression de ce fichier doit contenir la présente mention de copyright.

\section{cedram}




\title{
Perfect unary forms over real quadratic fields
}

\author{
par DAN YASAKI
}

\begin{abstract}
RÉsumÉ. Soit $F=\mathbb{Q}(\sqrt{d})$ un corps quadratique réel avec anneau d'entiers $\mathcal{O}$. Dans cet article, nous analysons le nombre $h_{d}$ de $\mathrm{GL}_{1}(\mathcal{O})$-orbites de classes d'homothétie des formes parfaites unaires sur $F$ en fonction de $d$. Nous calculons $h_{d}$ exactement pour $d \leq 200000$, sans carré. En reliant les formes parfaites aux fractions continues, nous donnons des bornes sur $h_{d}$ et répondons à certaines questions de Watanabe, Yano et Hayashi.
\end{abstract}

Abstract. Let $F=\mathbb{Q}(\sqrt{d})$ be a real quadratic field with ring of integers $\mathcal{O}$. In this paper we analyze the number $h_{d}$ of $\mathrm{GL}_{1}(\mathcal{O})$ orbits of homothety classes of perfect unary forms over $F$ as a function of $d$. We compute $h_{d}$ exactly for square-free $d \leq 200000$. By relating perfect forms to continued fractions, we give bounds on $h_{d}$ and address some questions raised by Watanabe, Yano, and Hayashi.

\section{Introduction}

The study of $n$-ary quadratic forms over the rational numbers is classical. Work of Minkowski and Voronoi brought a more geometric viewpoint, and the study of arithmetic minima of quadratic forms could be viewed as a study of lattice sphere packings. Perfect forms are a special class of positive definite quadratic forms that are uniquely determined by their minimal vectors and minimum. The natural action of $\mathrm{GL}_{n}(\mathbb{Z})$ on the space of positive definite quadratic forms preserves the set of perfect forms, and one wishes to understand the equivalence classes of perfect forms. For a nice survey of the literature, we refer the reader to [10].

These ideas have natural generalizations to number fields. Let $F$ be a totally real number field of degree $d$. Then $F$ has $d$ real embeddings. Identify the infinite places of $F$ with its embeddings. For each infinite place $v$ of $F$, let $V_{v}$ be the real vector space of $n \times n$ symmetric matrices $\operatorname{Sym}_{n}(\mathbb{R})$. Let $C_{v} \subset V_{v}$ be the corresponding cone of positive definite matrices. Set $V=\prod_{v} V_{v}$ and $C=\prod_{v} C_{v}$, where the products are taken over the infinite

Manuscrit reçu le 10 juillet 2012, révisé le 4 mars 2013.

Mots clefs. quadratic forms, perfect forms, continued fractions, real quadratic fields.

Classification math. 11E12. 
places of $F$. If $\alpha \in F$, let $\alpha_{v}$ denote its image in $F_{v}$. We extend this notation to matrices and vectors with coefficients in $F$.

One can think of $V$ as the space of positive definite $n$-ary quadratic forms over $F$ and the cone $C$ as the space of positive definite forms. Namely, if $A=\left(A_{v}\right) \in V$ and $x \in F^{n}$, then $A$ determines a quadratic form on $Q_{A}: F^{n} \rightarrow \mathbb{Q}$ by

$$
Q_{A}(x)=\sum_{v} x_{v}^{t} A_{v} x_{v}
$$

Note that if $A \in \operatorname{Sym}_{n}(F)$ is identified with its image $\left(A_{v}\right) \in V$, then

$$
Q_{A}(x)=\operatorname{Tr}_{F / \mathbb{Q}}\left(x^{t} A x\right) .
$$

Work of Koecher [4] generalizing Voronoi [12], allows one to compute perfect forms in this setting. Outside of explicit computation, however, not much is known about the number of perfect forms in $n$-variables, even for $F=\mathbb{Q}$. For $F$ a real quadratic field and $n=2$, examples of perfect forms has been computed in $[2,5,7]$. For $F$ a cyclotomic field and $n=1$, many computations have been done in [11]. Related ideas in a different context have been investigated in [1]. For a more general treatment of Voronoi reduction for $\operatorname{GL}\left(\Lambda_{0}\right)$, where $\Lambda_{0}$ is a projective $\mathcal{O}$-module, we refer the reader to [8] and a recent preprint of Watanabe, Yano, and Hayashi [13] for additional details and references.

In this paper, we consider perhaps the simplest non-trivial case where $F$ is a real quadratic field and $\Lambda_{0}=\mathcal{O}$. The main result given in Theorem 5.1, bounds the number of unary forms over $F$ in terms of period lengths of continued fractions by relating perfect forms with well-rounded binary quadratic forms. This gives an efficient method for computing perfect unary forms over $F$.

That paper is organized as follows. In Section 2, we set notation and given background on perfect unary forms over real quadratic fields. In Section 3, we recall some results about well-rounded quadratic forms and relate perfect unary forms over $F$ to well-rounded forms over $\mathbb{Q}$. In Section 4 , we collect some data plots of our computations of perfect unary forms for $F=\mathbb{Q}(\sqrt{d})$ for positive squarefree integers $d \leq 200000$. This consists of $10,732,735$ perfect forms divided among 121,580 fields. Finally, in Section 5, we give the main bound on the number of perfect forms by relating it to certain continued fractions and address some questions raised by Watanabe, Yano, and Hayashi [13].

Acknowledgments. I thank P. Gunnells and T. Watanabe for helpful discussions. I also thank the referee for many useful suggestions. 


\section{Background and notation}

In this section we fix notation for the real quadratic field $F$ and recall the notion of perfect unary forms in this case.

2.1. Real quadratic fields. Let $\mathcal{O}$ be ring of integers of the real quadratic field $F=\mathbb{Q}(\sqrt{d})$, where $d>0$ is a square-free integer. Let $F^{+} \subset F$ denote the totally positive elements of $F$. The fundamental discriminant of $F$ is

$$
D= \begin{cases}4 d & \text { if } d \equiv 2,3 \bmod 4 \\ d & \text { if } d \equiv 1 \bmod 4\end{cases}
$$

The ring of integers is $\mathcal{O}=\mathbb{Z}[\omega]$, where

$$
\omega= \begin{cases}\sqrt{d} & \text { if } d \equiv 2,3 \bmod 4, \\ \frac{1+\sqrt{d}}{2} & \text { if } d \equiv 1 \bmod 4\end{cases}
$$

Then $F$ has two real embeddings $v_{1}, v_{2}$.

2.2. Unary forms and perfection. Let $V=\mathbb{R}^{2}$, and let $C \subset V$ be the open cone $C=\mathbb{R}_{>0}^{2}$. Define trace $\operatorname{Tr}: V \rightarrow \mathbb{R}$ by

$$
\operatorname{Tr}\left(\left(A_{1}, A_{2}\right)\right)=A_{1}+A_{2} .
$$

Define addition and multiplication on $V$ componentwise. Using the real embeddings of $F$, we identify a point $x \in F$ with its image $\left(v_{1}(x), v_{2}(x)\right) \in$ $V$. By doing so, the arithmetic on $V$ is compatible with the arithmetic on $F$, and trace restricted to $F$ is just $\operatorname{Tr}_{F / \mathbb{Q}}$.

As described in Section 1, we view a point in $C$ as a positive definite quadratic form on $F=F^{1}$. Specifically, $A=\left(A_{1}, A_{2}\right) \in C$ determines a unary quadratic form $F \rightarrow \mathbb{R}$ by

$$
Q_{A}(x)=\operatorname{Tr}\left(A x^{2}\right)=A_{1} v_{1}\left(x^{2}\right)+A_{2} v_{2}\left(x^{2}\right) .
$$

We will write $A[x]$ for $Q_{A}(x)$.

The left action of $\mathrm{GL}_{1}(\mathcal{O})=\mathcal{O}^{\times}$on $V$ is given by

$$
g \cdot A=g A g^{t}=g^{2} A,
$$

where $g \in \mathrm{GL}_{1}(\mathcal{O})$ and $A \in V$. Note that since $g \in \mathrm{GL}_{1}(\mathcal{O}), g=g^{t}$ and multiplication is commutative. This action preserves $C \subset V$ and $F^{+} \subset C$.

Definition. Let $A \in C$ be a positive definite unary form over $F$. The minimum of $A$, denoted $m(a)$, is

$$
m(A)=\inf _{x \in \mathcal{O} \backslash\{0\}} A[x] .
$$

An element $\alpha \in \mathcal{O}$ is a minimal vector of $A$ if $A[\alpha]=m(A)$. 
Since $\mathcal{O}$ is discrete in $V$, for each $A$, the minimum $m(A)$ is attained by finitely many minimal vectors. Let $M(A)$ denote the set of minimal vectors for $A$.

Endow $V$ with the standard inner product $\langle\cdot, \cdot\rangle$ on $\mathbb{R}^{2}$. Define a map $q: \mathcal{O} \rightarrow V$ by $q(x)=x^{2}=\left(v_{1}\left(x^{2}\right), v_{2}\left(x^{2}\right)\right)$. Then for $A \in C$ and $x \in \mathcal{O}$,

$$
A[x]=\langle A, q(x)\rangle .
$$

In particular, each $x \in \mathcal{O}$ gives rise to a linear functional $q(x)$ on $V$.

Definition. A positive definite form $A \in C$ is perfect if

$$
\operatorname{span}_{\mathbb{R}}\{q(v) \mid x \in M(A)\}=V .
$$

From the definition, it is clear that a form is perfect if and only if it is uniquely determined by its minimum and its minimal vectors.

The following proposition is immediate from the definitions.

Proposition 2.1. Let $A \in C$ be a perfect form, and let $\lambda \in \mathbb{R}_{>0}$. Then

(1) $m(\lambda A)=\lambda m(A)$

(2) $M(\lambda A)=M(A)$

In particular, if $A$ is perfect, then $\lambda A$ is perfect.

Thus to classify perfect unary forms over $F$, it suffices to consider homothety classes of forms. Specializing work of Koecher [4] and Okuda-Yano [8] to this case, we get the following.

Proposition 2.2 ( $[4,8])$.

(1) There are finitely many $\mathrm{GL}_{1}(\mathcal{O})$-inequivalent homothety classes of perfect forms.

(2) If $A \in C$ is perfect, then there exists $\lambda \in \mathbb{R}_{>0}$ such that $\lambda A \in F^{+}$.

Let $h_{d}$ denote the number of $\mathrm{GL}_{1}(\mathcal{O})$-orbits of homothety classes of perfect unary forms. From Proposition 2.2, we know that $h_{d}<\infty$ for all $d$, and we wish to understand how $h_{d}$ behaves as we vary $d$.

\section{Unary forms over $F$ and binary forms over $\mathbb{R}$}

In this section, we reinterpret unary forms over $F$ as binary forms over $\mathbb{R}$. By doing so, we are able to identify the set perfect unary forms over $F$ as the intersection between a certain geodesic in the upper half plane $\mathfrak{h}$ and the trivalent tree $W$ of well-rounded forms shown in Figure (3.1).

3.1. Unary forms and $\mathfrak{h}$. $A \mathbb{Z}$-basis for $\mathcal{O}$ is given by $\mathcal{B}=\{1, \omega\}$, where $\omega$ is defined in (2.1). In terms of this basis, $A \in V$ can be viewed as a binary quadratic form corresponding to the symmetric matrix

$$
S_{A}=\left[\begin{array}{cc}
\operatorname{Tr}(A) & \operatorname{Tr}(A \omega) \\
\operatorname{Tr}(A \omega) & \operatorname{Tr}\left(A \omega^{2}\right)
\end{array}\right] .
$$


Specifically, for $x=a+b \omega$,

$$
A[x]=\left[\begin{array}{ll}
a & b
\end{array}\right] S_{A}\left[\begin{array}{l}
a \\
b
\end{array}\right] .
$$

We wish to study the forms up to real homothety (real positive scaling). If we scale $A$ so that $\operatorname{Tr}(A)=1$, then the corresponding matrix $S_{A}$ of the unary form $A$ has a particularly nice form. A straightforward computation shows the following.

Proposition 3.1. Let $A=s+t \omega \in C$ with $\operatorname{Tr}(A)=1$. Let $S_{A}$ denote the real $2 \times 2$ symmetric matrix corresponding to $A$. Then

$$
S_{A}=\left\{\begin{array}{lc}
{\left[\begin{array}{cc}
1 & 2 d t \\
2 d t & d
\end{array}\right]} & \text { if } d \equiv 2,3 \bmod 4 \\
{\left[\begin{array}{cc}
1 & (1+d t) / 2 \\
(1+d t) / 2 & (1+d+2 d t) / 4
\end{array}\right]} & \text { if } d \equiv 1 \bmod 4 .
\end{array}\right.
$$

We can identify the complex upper half-plane $\mathfrak{h}$ with positive definite symmetric matrices by

$$
x+i y \mapsto\left[\begin{array}{cc}
1 & -x \\
-x & x^{2}+y^{2}
\end{array}\right] .
$$

Up to scaling by positive homothety, this is consistent with the identification

$$
g \cdot i \mapsto\left(g g^{t}\right)^{-1}
$$

where $g \in \mathrm{SL}_{2}(\mathbb{Z})$.

Let $X_{\mathcal{B}}$ denote the image of $C$ modulo scaling in $\mathfrak{h}$ using Proposition 3.1, (3.1), and (3.3). Then a simple computation shows the following.

Proposition 3.2. The set $X_{\mathcal{B}}$ is the geodesic in $\mathfrak{h}$ defined by

$$
\begin{aligned}
x^{2}+y^{2} & =d & \text { if } d & \equiv 2,3 \bmod 4, \\
\left(x+\frac{1}{2}\right)^{2}+y^{2} & =\frac{d}{4} & \text { if } d & \equiv 1 \bmod 4 .
\end{aligned}
$$

3.2. Well-rounded binary forms. The well-rounded retract $W \subset \mathfrak{h}$ is the infinite trivalent tree shown in Figure 3.1. The tree $W$ represents the positive definite binary quadratic forms over $\mathbb{R}$ that are well-rounded. Recall that well-rounded forms are those binary quadratic forms whose minimal vectors span $\mathbb{R}^{2}$.

The well-rounded binary quadratic forms are completely understood. We collect some known facts about well-rounded forms. Let $\mathbb{P}^{1}(\mathbb{Q})=\mathbb{Q} \cup\{\infty\}$, with the usual convention that the cusp at $\infty$ is $1 / 0$.

Theorem 3.1. Identify the space of homothety classes of binary quadratic forms with $\mathfrak{h}$ using (3.4). Let $W \subseteq \mathfrak{h}$ denote the space of well-rounded forms. 


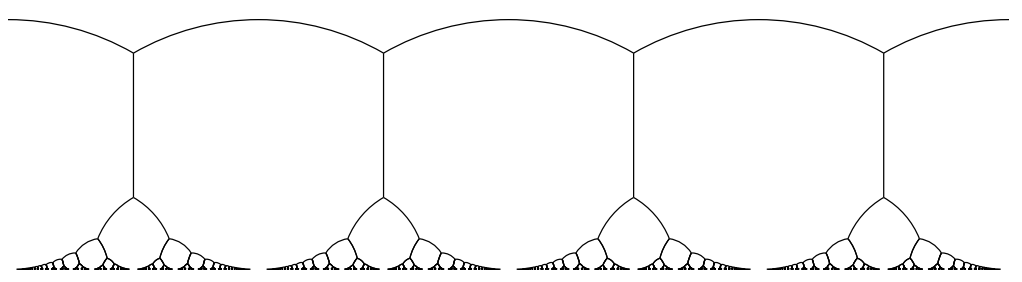

FiguRE 3.1. Well-rounded binary quadratic forms $W \subset \mathfrak{h}$

(1) There is a decomposition of $\mathfrak{h}$ into a disjoint union of sets

$$
\mathfrak{h}=W \cup \bigsqcup_{\alpha \in \mathbb{P}^{1}(\mathbb{Q})} H(\alpha),
$$

parameterized by points of $\mathbb{P}^{1}(\mathbb{Q})$. A binary form $\phi$ is in $H(\alpha)$ if and only if $M(\phi)=\left\{\left[\begin{array}{l}p \\ q\end{array}\right]\right\}$, where $\alpha=p / q$.

(2) For $\alpha \in \mathbb{P}^{1}(\mathbb{Q})$, let $W(\alpha) \subset W$ denote the boundary of $H(\alpha)$. Then

$$
W=\cup_{\alpha \in \mathbb{P}^{1}(\mathbb{Q})} W(\alpha) .
$$

A binary form $\phi \in W$ is in $W(\alpha)$ if and only if $\left[\begin{array}{l}p \\ q\end{array}\right] \in M(\phi)$, where $\alpha=p / q$.

(3) Let $\phi \in W$. If $\phi$ is perfect then $\phi$ is a vertex of $W$, and $\phi$ is $\mathrm{GL}_{2}(\mathbb{Z})$ equivalent to a binary form $\phi^{\prime}$ with

$$
M\left(\phi^{\prime}\right)=\left\{e_{1}, e_{2}, e_{1}+e_{2}\right\} .
$$

Otherwise, $\phi$ is $\mathrm{GL}_{2}(\mathbb{Z})$-equivalent to a binary form $\phi^{\prime}$ with

$$
M\left(\phi^{\prime}\right)=\left\{e_{1}, e_{2}\right\} \text {. }
$$

Remark. The term perfect in Theorem 3.1 refers to perfection of $\phi$ as a binary form over $\mathbb{Q}$. This is not to be confused with perfect unary forms over $F$. In particular, a perfect unary form over $F$ is not necessarily perfect as a binary form over $\mathbb{Q}$.

We now give a different characterization of perfect unary forms over $F$ by relating them to well-rounded binary forms.

Theorem 3.2. Let $A \in C$ be a unary form. Then $A$ is perfect if and only if $S_{A}$ is well-rounded.

Proof. First suppose $A \in C$ is perfect. Then there exists $\alpha, \beta \in M(A)$ such that $\left\{\alpha^{2}, \beta^{2}\right\}$ is linearly independent in $V$. It follows that $\{\alpha, \beta\}$ is linearly independent, and hence $S_{A}$ is well-rounded. 
Now suppose $S_{A}$ is well-rounded. Then $A$ has minimal vectors $\alpha$ and $\beta$ such that $\{\alpha, \beta\}$ is linearly independent in $V$. We wish to show that $\left\{\alpha^{2}, \beta^{2}\right\}$ is linearly independent. Suppose not. Then there exists $\lambda \in \mathbb{R}$ such that $\beta^{2}=\lambda \alpha^{2}$. Then $A[\beta]=\lambda A[\alpha]$. Since $\alpha$ and $\beta$ are minimal vectors of $A$, we have

$$
A[\alpha]=A[\beta]=m(A) .
$$

Then $\lambda=1$ and so $\beta= \pm \alpha$, which gives the desired contradiction.

Since the well-rounded binary forms over $\mathbb{R}$ have either two or three minimal vectors (up to sign), we immediately get the following.

Corollary 3.1. A perfect unary form over $F$ has either two or three (up to sign) minimal vectors.

By varying $d$, we see that both types of perfect unary forms arise. See Examples 5.4 and 5.4.

Voronoi's algorithm allows us to compute explicit representatives of the $\mathrm{GL}_{1}(\mathcal{O})$-equivalence classes of perfect unary forms given an initial input of a perfect unary form. From the explicit description of $X_{\mathcal{B}}$ and $W$, one can explicitly compute an initial perfect form.

Define $c_{d}$ to be

$$
c_{d}= \begin{cases}\sqrt{d} & \text { if } d \equiv 2,3 \bmod 4, \\ \frac{-1+\sqrt{d}}{2} & \text { if } d \equiv 1 \bmod 4 .\end{cases}
$$

It is easy to see that the geodesic $X_{\mathcal{B}}$ intersects the real axis at $c_{d}$.

Proposition 3.3. Let $x_{0}=\min \left\{X\left(\left\lfloor c_{d}\right\rfloor\right), X\left(\left\lfloor c_{d}\right\rfloor+1\right)\right\}$, where

$$
X(n)= \begin{cases}\frac{n^{2}+d-1}{2 n} & \text { if } d \equiv 2,3 \bmod 4, \\ \frac{4 n^{2}+d-5}{4+8 n} & \text { if } d \equiv 1 \bmod 4 .\end{cases}
$$

Let $n_{0} \in\left\{\left\lfloor c_{d}\right\rfloor,\left\lfloor c_{d}\right\rfloor+1\right\}$ satisfy $x_{0}=X\left(n_{0}\right)$. Define $A \in C$ to be

$$
A= \begin{cases}\frac{1}{2}-\frac{x_{0}}{2 d} \omega & \text { if } d \equiv 2,3 \bmod 4, \\ \frac{d+1+2 x_{0}}{2 d}-\frac{2 x_{0}+1}{d} \omega & \text { if } d \equiv 1 \bmod 4 .\end{cases}
$$

Then $A$ is a perfect unary form with $\left\{1, n_{0}+\omega\right\} \subseteq M(A)$.

Proof. The form $A$ is one of the intersection points of $X_{\mathcal{B}}$ with the top crenellation $W(\infty) \subseteq W$ consisting of portions of semicircles of radius 1 , centered at $(k, 0)$ for $k \in \mathbb{Z}$.

From Proposition 3.2, we see that $X_{\mathcal{B}}$ is a semicircle with center on the real axis and radius strictly larger than 1 . It follows that $X_{\mathcal{B}}$ intersects 
$W(\infty)$ exactly twice. We compute explicitly the intersection $x_{0}+i y_{0} \in \mathfrak{h}$ with $x_{0}>0$.

One can check that $X_{\mathcal{B}}$ intersects the semicircles

$$
(x-n)^{2}+y^{2}=1,
$$

for $n=\left\lfloor c_{d}\right\rfloor$ and $\left\lfloor c_{d}\right\rfloor+1$. Choose $n_{0} \in\left\{\left\lfloor c_{d}\right\rfloor,\left\lfloor c_{d}\right\rfloor+1\right\}$ such that the $x$ coordinate of the intersection $x_{0}+i y_{0}$ is smaller. This is the intersection of $X_{\mathcal{B}}$ with $W(\infty)$.

For $d \equiv 2,3 \bmod 4$, we compute the $x$-coordinate of the intersection of $x^{2}+y^{2}=d$ and $\left(x-n_{0}\right)^{2}+y^{2}=1$ to be

$$
x_{0}=\frac{n_{0}^{2}+d-1}{2 n_{0}} .
$$

From (3.3) and Proposition 3.1, we see that this intersection point corresponds to the perfect form $A=s+t \omega$ with $s=1 / 2$ and $t=-x_{0} /(2 d)$ as desired.

Similarly, for $d \equiv 1 \bmod 4$, the $x$-coordinate of the intersection of $(x+$ $1 / 2)^{2}+y^{2}=d / 4$ and $\left(x-n_{0}\right)^{2}+y^{2}=1$ is

$$
x_{0}=\frac{4 n_{0}^{2}+d-5}{4+8 n_{0}} \text {. }
$$

From (3.3) and Proposition 3.1, we see that this intersection point corresponds to the perfect form $A=s+t \omega$ with $s=\left(d+1+2 x_{0}\right) /(2 d)$ and $t=-\left(2 x_{0}+1\right) / d$ as desired.

From Theorem 3.1, $W(\infty) \cap W\left(n_{0}\right)$ is the set of well-rounded forms with $e_{1}$ and $n_{0} e_{1}+e_{2}$ as a minimal vector. Converting this to minimal vectors of the unary form $A$, we see that 1 and $n_{0}+\omega$ are minimal vectors of $A$.

Note that the perfect form $A$ constructed above may have more than just 1 and $n_{0}+\omega$ as minimal vectors. Specifically, when there are two integers $n$ such that $X(n)=x_{0}$, this gives rise to an additional minimal vector for $A$. In terms of the well-rounded tree, such a situation occurs when the geodesic $X_{\mathcal{B}}$ goes through a vertex of $W$.

Proposition 3.4. Let $u \in \mathcal{O}^{\times}$. Then there are exactly two homothety classes of perfect unary forms over $F$ that have $u$ as a minimal vector.

Proof. Note that the action of $u \in \mathrm{GL}_{1}(\mathcal{O})=\mathcal{O}^{\times}$on a quadratic unary form $A$ is given by

$$
u \cdot A=u^{2} A .
$$

It follows that the minimal vectors change by a factor of $u^{-1}$. Specifically,

$$
M(u \cdot A)=\left\{u^{-1} x \mid x \in M(A)\right\} .
$$




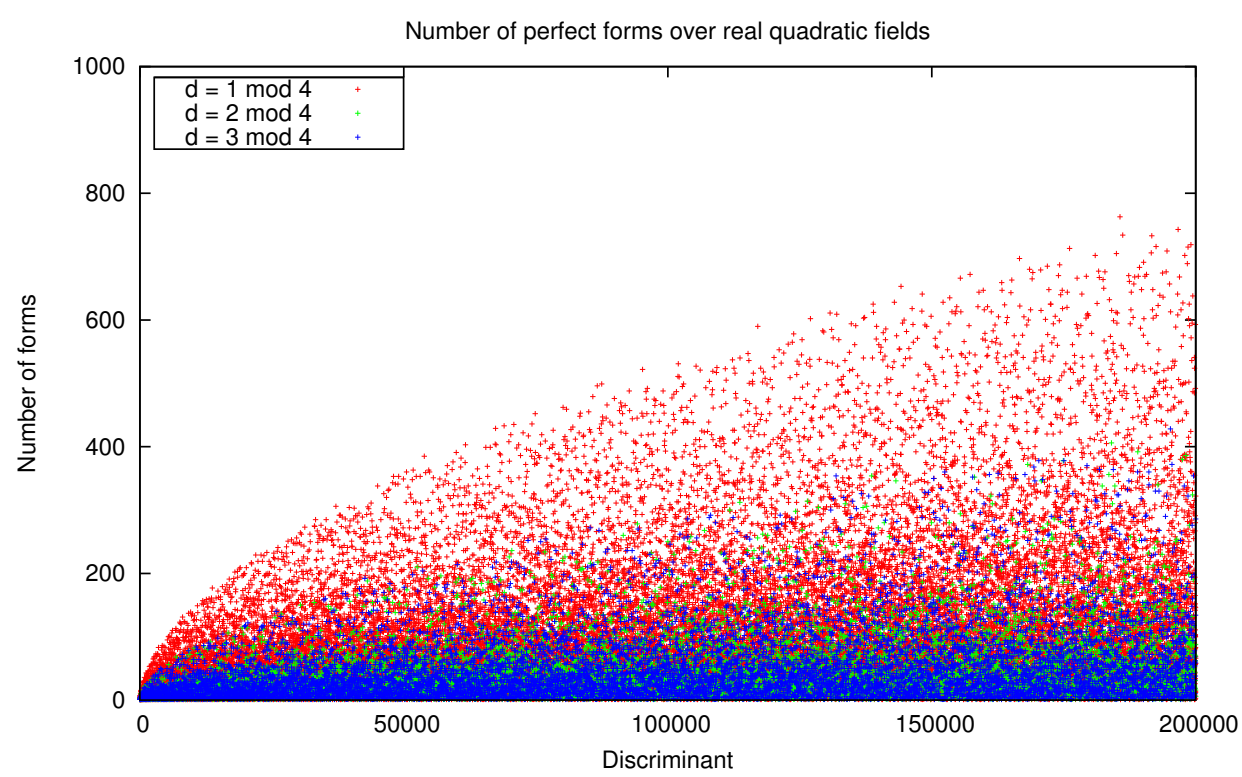

Figure 4.1. Number of $\mathrm{GL}_{1}(\mathcal{O})$-equivalence classes of perfect unary forms parameterized by discriminant of $F$

The construction in Proposition 3.3 shows there are exactly two perfect unary forms with 1 as a minimal vector. Acting on these forms with $\mathrm{GL}_{1}(\mathcal{O})$ gives the desired result.

\section{Data}

In this section, we collect some data plots of our computations of perfect unary forms. We explain these results by relating unary forms to continued fractions in Section 5 .

We first plot the number of forms by discriminant. See Figure 4 . Notice that on average there are far fewer perfect forms for discriminants that are $D=4 d$, where $d \equiv 2,3 \bmod 4$. Because of this, we compute more examples and parameterize by $d$. Indeed the different cases are much more similar when viewed as functions of $d$. See Figure 4 . The perfect forms for $F=\mathbb{Q}(\sqrt{d})$ are computed for $d \leq 200000$. This consists of $10,732,735$ perfect forms divided among 121,580 fields.

Next we sort the fields by the class number of the field. Specifically, we plot $h_{d}$ for $d \leq 200000$ with class number $h_{d} \leq 20$. The results are shown in Figure 4.3. Notice that the fields with large class number tend to have small number of perfect forms when compared to fields with small class number and similar size $d$. It is possible that this discrepancy can be explained if 


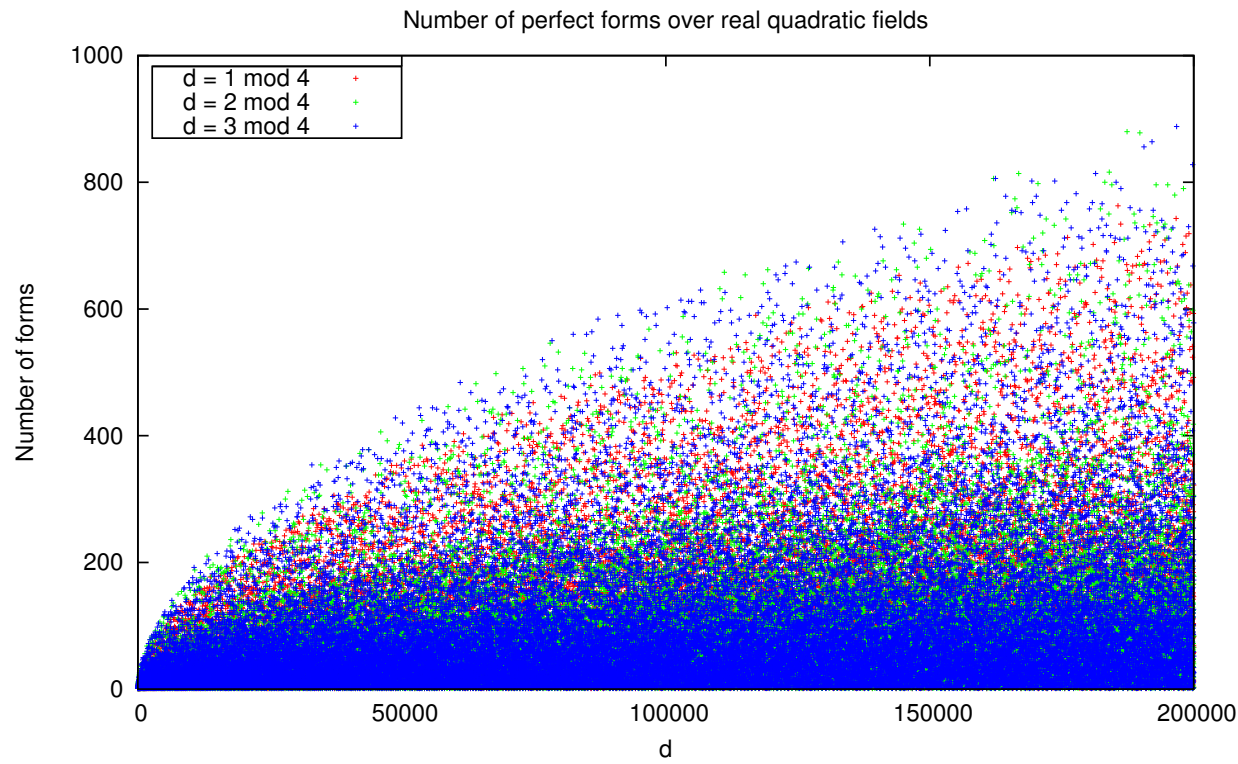

Figure 4.2. Number of $\mathrm{GL}_{1}(\mathcal{O})$-equivalence classes of perfect unary forms parameterized by $d$

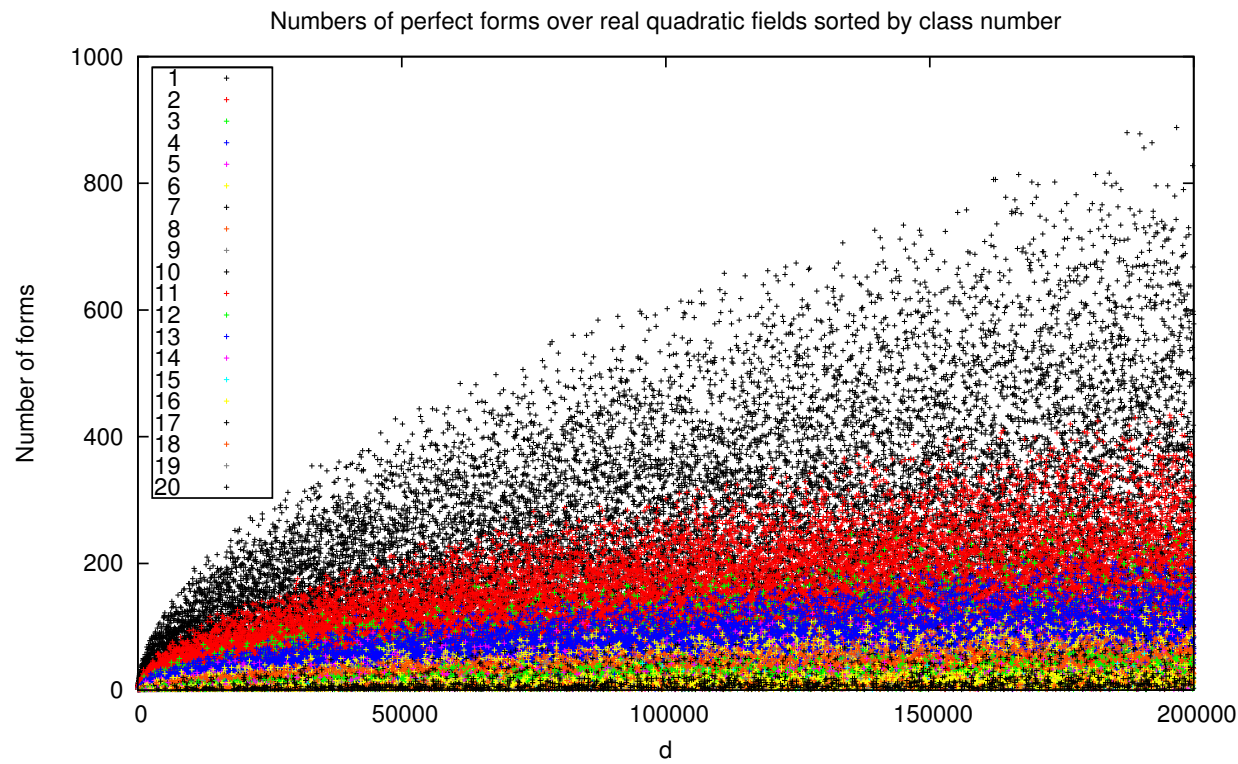

FiguRE 4.3. Number of perfect forms sorted by class number

one considers $\Lambda_{0}$-perfect forms, where $\Lambda_{0}$ ranges over representatives of the class group of $F$. We hope to address this in a future project. 


\section{Minimal vectors and continued fractions}

In this section, we describe a relationship between the minimal vectors of perfect unary forms and continued fractions. This yields a faster method of computing perfect unary forms.

5.1. Continued fractions. We set notation and recall known facts about continued fractions. A reference for the this material is [9].

Definition. A finite (simple) continued fraction is an expression of the form

$$
a_{0}+\frac{1}{a_{1}+\frac{1}{a_{2}+\frac{1}{\ddots+\frac{\ddots}{a_{n-1}+\frac{1}{a_{n}}}}},},
$$

where $a_{i} \in \mathbb{Z}$ for all $i$ and $a_{i}>0$ for $i>0$. We denote such an expression $\left[a_{0} ; a_{1}, \cdots, a_{n}\right]$.

Definition. The continued fraction $t_{k}=\left[a_{0} ; a_{1}, \ldots, a_{k}\right]$, where $0 \leq k \leq n$ is called the $k$ th convergent of the continued fraction $\left[a_{0} ; a_{1}, \ldots, a_{n}\right]$.

Using the Euclidean algorithm, one can see that every rational number can be written as a finite simple continued fraction. One can extend this theory to irrational numbers in the following way.

Proposition 5.1. Let $a_{0}, a_{1}, \ldots$ be an infinite sequence of integers with $a_{i}>0$ for $i>0$. Then the convergents $t_{k}$ tend to a limit $t$, that is,

$$
\lim _{k \rightarrow \infty} t_{k}=t .
$$

In this case, $t$ is called the value of the infinite simple continued fraction $\left[a_{0} ; a_{1}, \ldots\right]$, and we write $t=\left[a_{0} ; a_{1}, \ldots\right]$.

Proposition 5.2. Let $t=\alpha_{0}$ be an irrational number, and define the sequence $a_{0}, a_{1}, a_{2}, \ldots$ recursively by

$$
a_{k}=\left\lfloor\alpha_{k}\right\rfloor, \quad \alpha_{k+1}=\frac{1}{\alpha_{k}-a_{k}}
$$

for $k=0,1,2, \ldots$ Then $t=\left[a_{0} ; a_{1}, a_{2}, \ldots\right]$.

Proposition 5.3 ( $[9$, Theorems 12.10, 12.11, 12.21]). Let $t$ be a real number with convergents $t_{k}=p_{k} / q_{k}$ in reduced form. Then

(1) $t_{k} \geq t$ for $k$ odd and $t_{k} \leq t$ for $k$ even.

(2) $\operatorname{det}\left(\left[\begin{array}{ll}p_{i} & p_{i+1} \\ q_{i} & q_{i+1}\end{array}\right]\right) \in\{ \pm 1\}$. 
(3) The continued fraction expansion of $t$ is periodic if and only if $t$ is a quadratic irrational.

Remark. Let $\left[a_{0} ; a_{1}, \ldots\right]$ be the continued fraction expansion of $t \in \mathbb{R}$. The convergents form a sequence of rational numbers converging to $t$. This can be interpreted as giving a sequence of cusps in $\mathbb{P}^{1}(\mathbb{Q})$, where $p / q$ is identified with the line through $(p, q)$.

Proposition 5.4. Fix a positive, square-free integer $d$. Let $c_{d}$ be defined as in (3.5), and let $\omega$ be defined as in (2.1). Let $\ell_{d}$ denote the period of the continued fraction for $c_{d}$. Then $p+q \omega$ is the fundamental unit for $\mathbb{Q}(\sqrt{d})$, where $p / q$ is the $\left(\ell_{d}-1\right)$-th convergent of $c_{d}$, written in reduced form.

5.2. Minimal vectors of perfect unary forms. Let $A_{1}$ denote the perfect form from Proposition 3.3 with minimal vectors including 1 and $n+\omega$. From Proposition 3.4, there are two perfect forms with minimal vector 1 . Continuing along the geodesic away from the other perfect form, we come to another intersection with $W$. Hence we obtain another perfect form $A_{2}$. We continue in this manner until we find a form $A_{n+1} \in\left\{\epsilon^{2} A_{1}, \epsilon^{-2} A_{1}\right\}$, where $\epsilon$ is the fundamental unit of $\mathcal{O}$. Then $\left\{A_{1}, \cdots, A_{n}\right\}$ form a complete set of representatives for the $\mathrm{GL}_{1}(\mathcal{O})$-conjugacy classes of perfect forms over $F$.

Adjacent forms $A_{i}$ and $A_{i+1}$ share exactly one minimal vector. Thus there is a well-defined sequence of elements of $\mathcal{O}$ associated to $\left\{A_{1}, \cdots, A_{n}\right\}$ given by the ordered list of minimal vectors, deleting repeats for the shared minimal vectors. Each element $s+t \omega \in \mathcal{O}$ can be identified with a cusp $s / t \in \mathbb{P}^{1}(\mathbb{Q})$. Thus there is a well-defined sequence of cusps associated to each sequence of representative perfect forms. Each cusp $\alpha \in \mathbb{P}^{1}(\mathbb{Q})$ corresponds to a connected component $H(\alpha)$ as described in Theorem 3.1. Other than the first cusp $\infty$, the remaining cusps can be identified with elements of $\mathbb{Q}$. It is this sequence of cusps that is related to convergents of $c_{d}$, where $c_{d}$ is defined in (3.5). This relationship is made precise in the proof of Theorem 5.1.

\subsection{Bounding $h_{d}$.}

Theorem 5.1. Let $h_{d}$ be the number of $\mathrm{GL}_{1}(\mathcal{O})$-orbits of homothety classes of perfect unary forms over $F$, and let $\ell_{d}$ denote the period length of $c_{d}$. Then

$$
\frac{\ell_{d}}{2} \leq h_{d} \leq \ell_{d}
$$

Proof. We identify unary forms over $F$ with binary quadratic forms over $\mathbb{R}$ and identify cusps with minimal vectors as described in Section 3 . In 
particular, a cusp $p / q \in \mathbb{Q}$ is identified with $p+q \omega \in \mathcal{O}$ and $\left[\begin{array}{l}p \\ q\end{array}\right] \in \mathbb{Z}^{2}$, and the cusp $\infty$ is identified with $1 \in \mathcal{O}$ and $\left[\begin{array}{l}1 \\ 0\end{array}\right] \in \mathbb{Z}^{2}$.

From Theorem 3.2, it suffices to understand the intersection of the geodesic $X_{\mathcal{B}}$ defined in Proposition 3.2 and the well-rounded binary quadratic forms $W$ shown in Figure 3.1. To this end, we consider the Farey tessellation [3]. This is a tessellation of $\mathfrak{h}$ by ideal triangles that is dual to the tree of well-rounded binary quadratic forms. For each vertex of $W$, there is a triangle with vertices in $\mathbb{P}^{1}(\mathbb{Q})$, and two triangles meet along an edge if and only if there is an edge in $W$ joining the corresponding vertices. See Figure 5.1.

Let $\Delta$ be an ideal triangle of the tessellation with vertices $\left\{\alpha_{1}, \alpha_{2}, \alpha_{3}\right\}$. Then $W$ divides $\Delta$ into three regions, and we can write $\Delta$ as

$$
\Delta=\Delta_{W} \sqcup \Delta_{1} \sqcup \Delta_{2} \sqcup \Delta_{3},
$$

where $\Delta_{W}=\Delta \cap W$ and $\Delta_{i}=\Delta \cap H\left(\alpha_{i}\right)$ for $i=1,2,3$.

Starting with the initial perfect unary form $A_{1}$, there is a sequence of adjacent perfect unary forms $\left\{A_{1}, \cdots, A_{n}\right\}$ that form a complete set of representatives for the $\mathrm{GL}_{1}(\mathcal{O})$-conjugacy classes. Let $A_{0}$ denote the perfect form that is adjacent to $A_{1}$ that shares the minimal vector 1 . Let $t=$ $\left\{t_{k}\right\}_{k=-1}^{\infty}$ denote the sequence of convergents of $c_{d}$, identified with cusps and minimal vectors as described above, where we set $t_{-1}=\infty$. Let $\left\{\alpha_{1}, \alpha_{2}, \alpha_{3}\right\}$ be a subsequence of consecutive elements of $t$, where $\alpha_{1}$ corresponds to a minimal vector shared by two adjacent unary forms $A_{k}$ and $A_{k+1}$ in $\left\{A_{0}, \ldots, A_{n}\right\}$. For example, the minimal vector shared by $A_{0}$ and $A_{1}$ is 1 , which corresponds to cusp $\infty$. Then $\alpha_{1}=\infty, \alpha_{2}=t_{0}$, and $\alpha_{3}=t_{2}$. We will show in general that $\left\{\alpha_{1}, \alpha_{i}\right\} \subseteq M\left(A_{k+1}\right)$ for $i=2$ or $i=3$.

Since $\alpha_{1}$ corresponds to a minimal vector shared by $A_{k}$ and $A_{k+1}$, the geodesic $X_{\mathcal{B}}$ intersects $\Delta_{1}$ of the decomposition in (5.1) non-trivially. Furthermore, since $c_{d}$ is between $\alpha_{2}$ and $\alpha_{3}$ and $X_{\mathcal{B}}$ terminates at $c_{d}, X_{\mathcal{B}}$ must intersect the geodesic joining $\alpha_{2}$ and $\alpha_{3}$. It follows that $X_{\mathcal{B}}$ intersects $\Delta_{i}$ non-trivially for $i=2$ or $i=3$. Since $X_{\mathcal{B}}$ is a continuous path, $X_{\mathcal{B}}$ must intersect $\Delta_{W}$. This intersection point $\Delta_{W} \cap X_{\mathcal{B}}$ corresponds to $A_{k+1}$, showing that $\alpha_{2}$ or $\alpha_{3}$ is a minimal vector of $A_{k+1}$.

Therefore, the minimal vectors arising in the adjacent forms $\left\{A_{1}, \ldots A_{n}\right\}$ can be identified with a subsequence of the cusps $t=\left\{\infty, t_{1}, t_{2}, \ldots\right\}$ coming from the convergents of $c_{d}$. We are counting perfect forms up to the action $\mathrm{GL}_{1}(\mathcal{O})$, which scales the minimal vectors of a perfect form by a unit. Thus $h_{d} \leq \ell_{d}$. Furthermore, the argument above shows that this subsequence of minimal vectors cannot miss two consecutive terms of $t$. Then $\ell_{d} \leq 2 h_{d}$, and the result follows. 


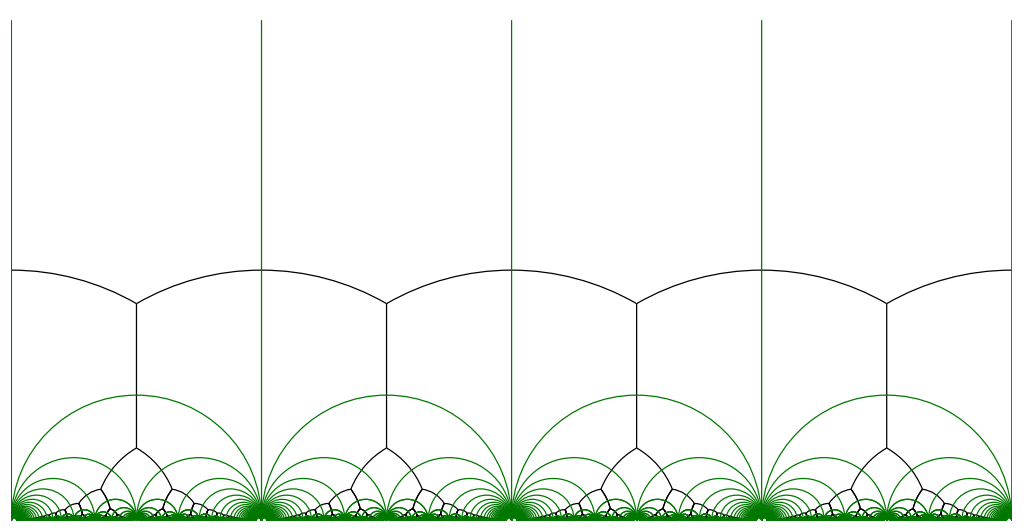

FiguRE 5.1. Well-rounded binary quadratic forms (black) with dual tessellation by ideal triangles (green)

Watanabe, Yano, and Hayashi [13] pose two questions about the nature of $h_{d}$.

(1) Are there infinitely many $d$ such that $h_{d}=1$ ?

(2) Is $h_{d}$ unbounded?

We answer both of these in the affirmative using our bounds in Theorem 5.1.

Corollary 5.1. There are infinitely many squarefree $d>1$ such that $h_{d}=1$.

Proof. By Theorem 5.1, it suffices to find an infinite family of squarefree $d$ such that $\ell_{d}=1$.

Let $f(x) \in \mathbb{Z}[x]$ be an irreducible quadratic polynomial. Let

$$
N_{f}=\operatorname{gcd}(f(0), f(1), f(2))
$$

Nagel [6] shows that if $N_{f}$ is squarefree, then there are infinitely many integers $n$ such that $f(n)$ is squarefree.

Let $f(x)=2 x^{2}+2 x+1$, and let $S=\{f(n) \mid n \in \mathbb{Z}, f(n)$ squarefree $\}$. One checks that $N_{f}=1$, and so by Nagel's result, $S$ contains infinitely many integers. Furthermore, since $S$ consists of odd integers, $T=\{2 s \mid s \in S\}$ is also infinite. The set $T$ provides the desired family. Namely, for $d \in T$, we have $d \equiv 2 \bmod 4$, and so $c_{d}=\sqrt{d}$. The continued fraction expansion of $c_{d}$ in this case is $c_{d}=[a, \overline{2 a}]$, where $a=\lfloor\sqrt{d}\rfloor$. Since $\ell_{d}=1$ for every $d \in T$, Theorem 5.1 gives the desired result. 


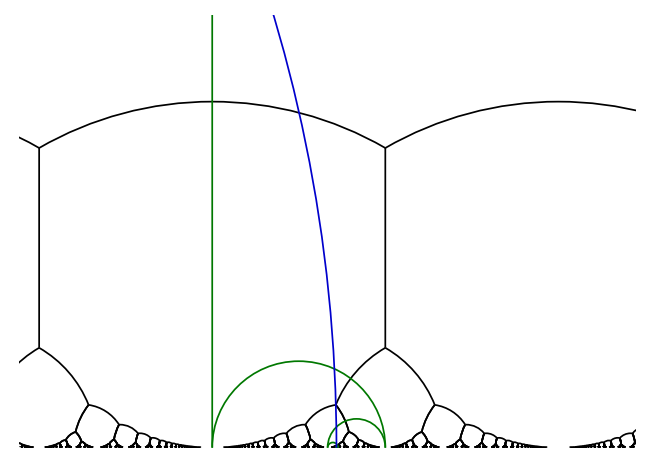

Figure 5.2. Geodesic $X_{\mathcal{B}}$ (blue) and well-rounded retract $W$ (black) for $F=\mathbb{Q}(\sqrt{19})$ with the sequence of geodesics (green) associated to the continued fraction expansion of $c_{19}$

Remark. In our computations of the exact values of $h_{d}$ for squarefree positive $d<200000$, we found four disjoint families with $h_{d}=1$. They are

$$
\begin{aligned}
& T_{1}=\left\{n^{2}+1: n^{2}+1 \text { is square free and } n \text { is odd }\right\}, \\
& T_{2}=\left\{n^{2}-1: n^{2}-1 \text { is square free and } n \text { is even }\right\}, \\
& T_{3}=\left\{n^{2}+4: n^{2}+4 \text { is square free and } n \text { is odd }\right\}, \text { and } \\
& T_{4}=\left\{n^{2}-4: n^{2}-4 \text { is square free and } n \text { is odd, } n>3\right\} .
\end{aligned}
$$

The set $T_{1}$ is the family given in the proof of Corollary 5.1.

We next consider the question about the unboundedness of $h_{d}$. From the lower bound in Theorem 5.1, to show that $h_{d}$ is unbounded, it suffices to produce infinite families with $\ell_{d}$ unbounded. Indeed there are explicit infinite families of $d$ such that period length of the continued fraction expansion of $c_{d}$ grows without bound. Thus by Theorem $5.1, h_{d}$ is unbounded.

\subsection{Examples.}

Example. Let $F=\mathbb{Q}(\sqrt{19})$, and let $\mathcal{O} \subset F$ be its ring of integers. Then $\mathcal{O}=\mathbb{Z}[\omega]$, where $\omega=\sqrt{19}$. Then $F$ has class number 1 and narrow class number 2 . There are four $\mathrm{GL}_{1}(\mathcal{O})$-equivalence classes of perfect unary forms $\left\{A_{1}, A_{2}, A_{3}, A_{4}\right\}$. The minimal vectors are

$$
\begin{aligned}
& M\left(A_{1}\right)=\{1, \omega+4\} \\
& M\left(A_{2}\right)=\{\omega+4,2 \omega+9,3 \omega+13\} \\
& M\left(A_{3}\right)=\{3 \omega+13,11 \omega+48,14 \omega+61\} \\
& M\left(A_{4}\right)=\{14 \omega+61,39 \omega+170\}
\end{aligned}
$$


which gives rise to the sequence of cusps

$$
\frac{1}{0}, \frac{4}{1}, \frac{9}{2}, \frac{13}{3}, \frac{48}{11}, \frac{61}{14}, \frac{170}{39} .
$$

The continued fraction expansion of $c_{19}=\sqrt{19}$ is

$$
c_{19}=[4 ; \overline{2,1,3,1,2,8}] \text {. }
$$

The convergents are

$$
4, \frac{9}{2}, \frac{13}{3}, \frac{48}{11}, \frac{61}{14}, \frac{170}{39}, \ldots
$$

In this case we see that the convergents of $c_{19}$ correspond exactly to the minimal vectors of perfect unary forms over $F=\mathbb{Q}(\sqrt{19})$.

Next we examine a case where the sequence of minimal vectors is a proper subsequence of the convergents.

Example. Let $F=\mathbb{Q}(\sqrt{23})$, and let $\mathcal{O} \subset F$ be its ring of integers. Then $\mathcal{O}=\mathbb{Z}[\omega]$, where $\omega=\sqrt{23}$. Then $F$ has class number 1 and narrow class number 2. There are two $\mathrm{GL}_{1}(\mathcal{O})$-classes of perfect unary forms $\left\{A_{1}, A_{2}\right\}$. The minimal vectors of the form are

$$
\begin{aligned}
& A_{1}=\{1, \omega+5\} \\
& A_{2}=\{\omega+5,5 \omega+24\}
\end{aligned}
$$

which gives rise to the sequence of cusps

$$
\frac{1}{0}, \frac{5}{1}, \frac{24}{5} \text {. }
$$

We compute the continued fraction expansion of $c_{23}=\sqrt{23}$ and find

$$
\sqrt{23}=[4 ; \overline{1,3,1,8}] \text {. }
$$

The convergents are

$$
4,5, \frac{19}{4}, \frac{24}{5}, \ldots
$$

In this case, the sequence of cusps corresponding to minimal vectors is a proper subsequence of the convergents.

\section{References}

[1] Eva Bayer-Fluckiger And Gabriele Nebe, On the Euclidean minimum of some real number fields. J. Théor. Nombres Bordeaux, 17(2) (2005), 437-454.

[2] Paul E. Gunnells and Dan Yasaki, Hecke operators and Hilbert modular forms. In Algorithmic number theory, volume 5011 of Lecture Notes in Comput. Sci., pages 387-401. Springer, Berlin, 2008.

[3] A. HuRwitz, Ueber die Reduction der binären quadratischen Formen. Math. Ann., 45(1) (1894), 85-117.

[4] Max Koecher, Beiträge zu einer Reduktionstheorie in Positivitätsbereichen. I. Math. Ann., 141 (1960), 384-432.

[5] Alar LEIBAK, The complete enumeration of binary perfect forms over the algebraic number field $\mathbb{Q}(\sqrt{6})$. Proc. Estonian Acad. Sci. Phys. Math., 54(4) (2005), 212-234. 
[6] Trygve NAgel, Zur arithmetik der polynome. Abhandlungen aus dem Mathematischen Seminar der Universität Hamburg, 1 (1922), 178-193. 10.1007/BF02940590.

[7] Heidrun E. Ong, Perfect quadratic forms over real-quadratic number fields. Geom. Dedicata, 20(1) (1986), 51-77.

[8] Kenji Okuda and Syouji Yano, A generalization of Voronoï's theorem to algebraic lattices. J. Théor. Nombres Bordeaux, 22(3) (2010), 727-740.

[9] Kenneth H. Rosen, Elementary number theory and its applications. Pearson, Reading, MA, sixth edition, 2010.

[10] Achill Schürmann, Enumerating perfect forms. In Quadratic forms-algebra, arithmetic, and geometry, volume 493 of Contemp. Math., pages 359-377. Amer. Math. Soc., Providence, RI, 2009.

[11] FrançOIs Sigrist, Cyclotomic quadratic forms. J. Théor. Nombres Bordeaux, 12(2) (2000), 519-530. Colloque International de Théorie des Nombres (Talence, 1999).

[12] G. Voronoř, Sur quelques propriétés des formes quadratiques positives parfaites. J. Reine Angew. Math., 133 (1908), 97-178.

[13] Takao Watanabe, Syouji Yano, and Takuma Hayashi, Voronoï's reduction theory of $G L_{n}$ over a totally real field. Preprint at http://www.math.sci.osaka-u.ac.jp/ twatanabe/ voronoireduction.pdf.

\section{Dan YASAKI}

Department of Mathematics and Statistics

The University of North Carolina at Greensboro

Greensboro, NC 27412, USA

E-mail: d_yasaki@uncg.edu

URL: http://www.uncg.edu/ d_yasaki/ 